\title{
„Kinderneuroradiologie braucht eine bessere Vernetzung“
}

Dr. med. Monika Huhndorf ist angehende Neuroradiologin am Universitätsklinikum Schleswig-Holstein, Campus Kiel. Gemeinsam mit Vertreterinnen der Gesellschaft für Pädiatrische Radiologie hat sie ein Expertennetzwerk Neuropädiatrische Bildgebung ins Leben gerufen, das den Austausch von Bildbefunden und die Diskussion im Kreis von Fachleuten erleichtern soll. Das Netzwerk setzt dabei auf eine DSGVO-konforme Messenger-Software.

Frau Dr. Huhndorf, was hat Sie zur Gründung eines Neuropädiatrischen Netzwerks bewogen?

Aus meiner Sicht einer angehenden Neuroradiologin stellt die pädiatrische Neuroradiologie ein großes Feld zwischen Neuroradiologie und Kinderradiologie dar. Eine gute diagnostische Qualität auf diesem vielseitigen Gebiet erfordert, wie in allen Spezialbereichen der Radiologie, jahrelange Expertise und Liebe zum Detail. Die Sonderstellung der pädiatri- schen Neuroradiologie zwischen Neuroradiologie und Kinderradiologie führt dazu, dass dieser Bereich in Deutschland nur wenig Sichtbarkeit erfährt. Nationale Fortbildungsveranstaltungen sind rar und die Vernetzungsmöglichkeiten der Kolleginnen und Kollegen, die sich mit diesem Thema beschäftigen, hierdurch eingeschränkt.

Während insbesondere die Neuroradiologie in den letzten Jahren auch zahlenmäßig einen großen Aufschwung erlebt hat, gibt es auch innerhalb großer neuroradiologischer Abteilungen erfahrungsgemäß nur ein bis zwei Radiologinnen oder Radiologen, die sich vermehrt der Kinderneuroradiologie angenommen haben. Bei kniffligen Fällen, wie sie einem in der Kinderneuroradiologie regelmäßig begegnen, ist der kollegiale Austausch vor Ort daher begrenzt.

Ich selbst beschäftige mich seit etwas mehr als einem Jahr intensiver mit diesem Fachbereich und bin durch Gespräche mit unserem Klinikdirektor Prof. Jansen auf die Idee

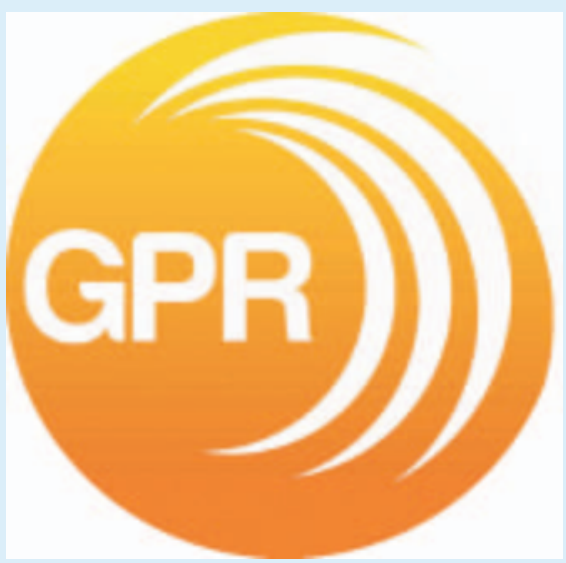

gebracht worden, eine bessere Vernetzung und Sichtbarkeit der Kinderneuroradiologie in Deutschland anzustoßen.

\section{An wen richtet sich das Netzwerk? Wen möchten Sie ansprechen?}

Das Netzwerk richtet sich an alle radiologischen Kolleginnen und Kollegen, die Interesse an pädiatrischer Neuroradiologie haben. Ich 
wünsche mir einen lebhaften Austausch zwischen jungen und erfahrenen Radiologinnen und Radiologen auf diesem Gebiet.

Welche Funktionen soll das Netzwerk erfüllen?

In meiner Vision soll das Netzwerk gleich mehrere Aufgaben erfüllen: Ein wichtiger Punkt ist die Möglichkeit einer Falldiskussion. Innerhalb des Netzwerkes soll man sowohl die Möglichkeit haben, sich einen diagnostischen Rat von Erfahrenen einzuholen, als auch bereits gelöste und lehrreiche Fälle mit der Gruppe zu teilen, um gleichzeitig einen Fortbildungscharakter zu gewährleisten. Genauso würde ich mir wünschen, dass interessante und diagnostisch relevante Literatur miteinander geteilt werden kann. Damit meine ich einen Austausch über neue Publikationen und auch mal einen schönen Übersichtsartikel, der im Klinikalltag hilfreich sein kann. Das Wissen aller Kolleginnen und Kollegen sollte sich hier bündeln und so die diagnostische Versorgung der Patientinnen und Patienten verbessern.

Wie der Name schon sagt, ist natürlich auch das „Netzwerken“ eine der Hauptaufgaben. Um die Sichtbarkeit der Kinderneuroradiologie in Deutschland zu verbessen und für diese relativ kleine Zielgruppe von Interessierten Fortbildungsmöglichkeiten zu schaffen, ist es wichtig, dass wir uns vernetzen, um als Gruppe auftreten zu können. Vielleicht ergeben sich sogar Forschungskooperationen.
Das Netzwerk nutzt eine datenschutzkonforme Messanger-App. Welche Funktionen stehen hier zur Verfügung und welche Vorteile bieten diese?

Die App heißt siilo und bietet die Möglichkeit, medizinische Daten DSGVO-konform auszutauschen. Wir sind in einer Zeit angekommen, in der der Griff zum Smartphone zum Schreiben einer Nachricht in einer Messenger App häufig schneller geht, als eine E-Mail zu schreiben. Dies gilt insbesondere, wenn die Nachricht eine große Gruppe erreichen soll. Siilo bietet neben der üblichen Chatfunktion die Möglichkeit innerhalb eines vertrauten Netzwerkes zu kommunizieren.

Diese Form der Kommunikation über Siilo bietet eine Organisationsstruktur mit unterschiedlichen Ordnern. So können beispielsweise Falldiskussionen von organisatorischen Dingen getrennt werden. Darüber hinaus bietet die Falldiskussion eine Diskussion innerhalb eines eigenen Chatraumes für jeden Fall, sodass innerhalb einer interaktiven Diskussion klar ist, auf welchen Fall sich die Kommentare beziehen - anders als in großen Gruppenchats.

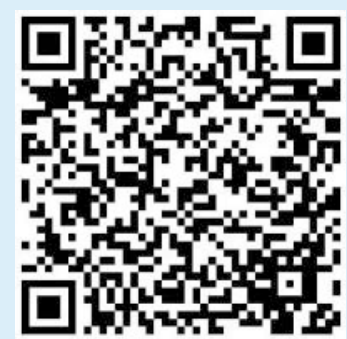

Wenn Sie die App von siilo bereits nutzen, gelangen Sie mit diesem QR-Code direkt in das Netzwerk Neuropädiatrische Radiologie.
Welche Fachgesellschaften beteiligen sich an dem Projekt?

Erfreulicherweise ist das Projekt innerhalb der radiologischen Fachgesellschaften auf positive Resonanz gestoßen. So ist es uns möglich, dieses Projekt unter dem Dach der Deutschen Röntgengesellschaft mit Unterstützung der Deutschen Gesellschaft für Neuroradiologie und der Gesellschaft für Pädiatrische Radiologie anzusiedeln.

\section{Wie kann man mitmachen?}

Mitmachen ist ganz einfach für alle Kolleginnen und Kollegen, die sich für Kinderneuroradiologie interessieren. Die App siilo ist kostenfrei herunterzuladen. Zugangsdaten gibt es auch in der Geschäftsstelle (Florian Schneider, Referent Tel. 030/916 07019 oder E-Mail: schneider@drg.de).Wir werden in den kommenden Wochen auch noch einen Kreis an Kolleginnen und Kollegen direkt anschreiben.

Vielen Dank für das Gespräch! 